\title{
Effect of Pressure on Interband and Intraband Transition of Mercury Chalcogenides Quantum Dots
}

Clément Livache ${ }^{1,2}$, Nicolas Goubet ${ }^{1,2}$, Charlie Gréboval ${ }^{1}$, Bertille Martinez ${ }^{1,2}$, Julien Ramade ${ }^{1}$, Junling Qu ${ }^{1}$, Amaury Triboulin ${ }^{1}$, Hervé Cruguel ${ }^{1}$, Benoit Baptiste ${ }^{3}$, Stefan Klotz ${ }^{3}$, Guy Fishman ${ }^{4}$, Sébastien Sauvage ${ }^{4}$, Francesco Capitani ${ }^{5}$, Emmanuel Lhuillier ${ }^{1 *}$

${ }^{1}$ Sorbonne Université, CNRS, Institut des NanoSciences de Paris, INSP, F-75005 Paris, France

'Laboratoire de Physique et d'Etude des Matériaux, ESPCI-ParisTech, PSL Research University, Sorbonne Université UPMC Univ Paris 06, CNRS, 10 rue Vauquelin 75005 Paris, France.

${ }^{3}$ Sorbonne Université, CNRS, Institut de Minéralogie, de Physique des Matériaux et de Cosmochimie, IMPMC, F-75005 Paris, France

${ }^{4}$ Centre de Nanosciences et de Nanotechnologies, CNRS, Univ. Paris-Sud, Université ParisSaclay, C2N - 10 boulevard Thomas Gobert, 91460 Palaiseau, France

${ }^{5}$ Synchrotron-SOLEIL, Saint-Aubin, BP48, F91192 Gif sur Yvette Cedex, France

To whom correspondence should be sent: el@insp.upmc.fr

\section{Table of content}

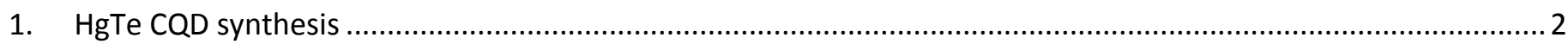

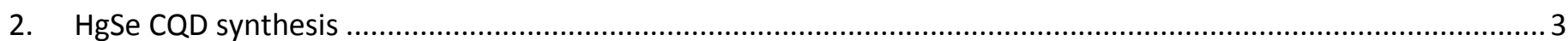

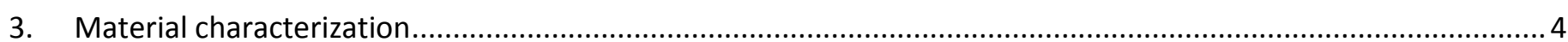

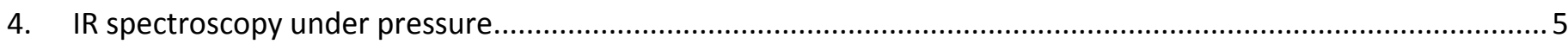

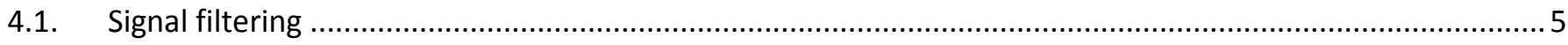

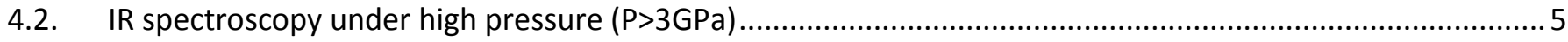

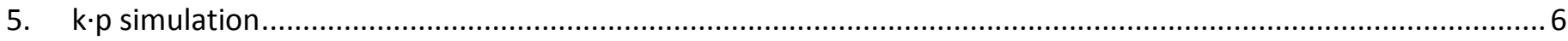

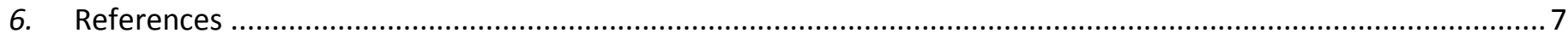




\section{HgTe $6000 \mathrm{~cm}^{-1}$}

HgTe $4000 \mathrm{~cm}^{-1}$

HgTe $2000 \mathrm{~cm}^{-1}$
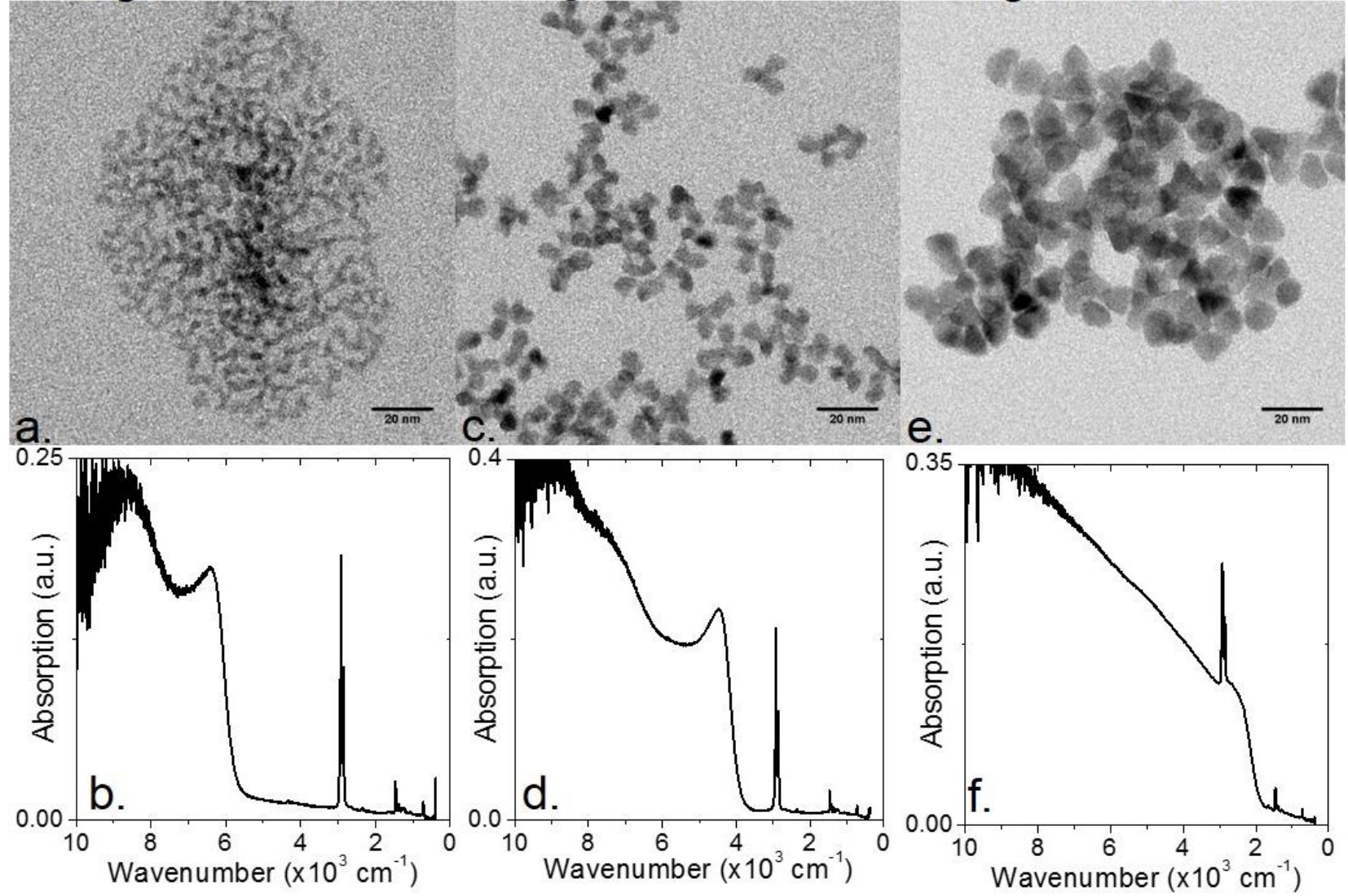

Figure $S 1 a$ and $b$ are respectively TEM image and zero pressure infrared spectrum from HgTe CQD with a band edge energy at $6000 \mathrm{~cm}^{-1}$. c and d are respectively TEM image and zero pressure infrared spectrum from HgTe CQD with a band edge energy at $4000 \mathrm{~cm}^{-1}$. e and $f$ are respectively TEM image and zero pressure infrared spectrum from HgTe CQD with a band edge energy at $2000 \mathrm{~cm}^{-1}$ 


\section{$\mathrm{HgSe} 3000 \mathrm{~cm}^{-1}$}

$\mathrm{HgSe} 2500 \mathrm{~cm}^{-1}$

$\mathrm{HgSe} 1000 \mathrm{~cm}^{-1}$
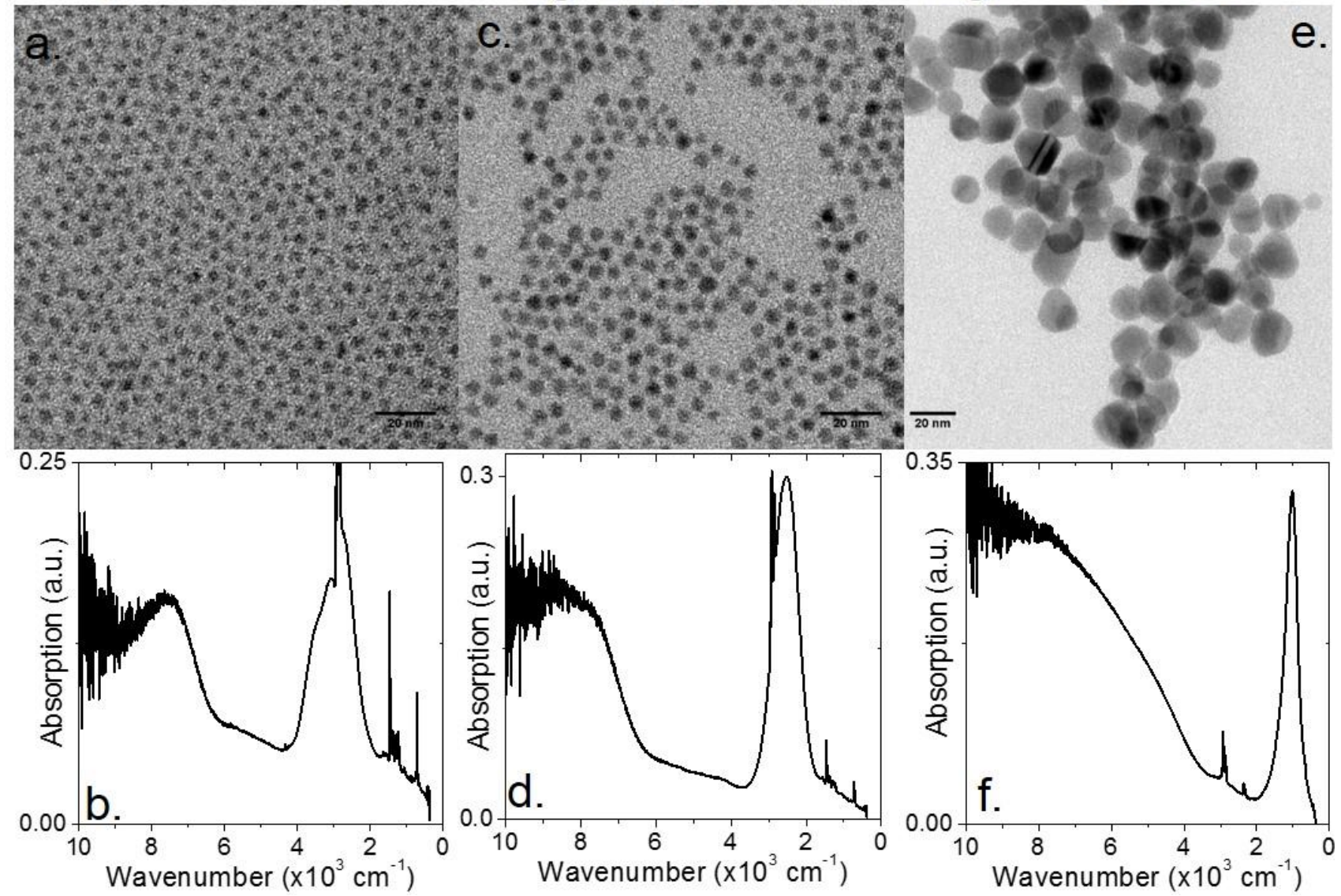

Figure $S 2 a$ and $b$ are respectively TEM image and zero pressure infrared spectrum from HgSe CQD with an intraband peak energy at $3000 \mathrm{~cm}^{-1} . c$ and d respectively TEM image and zero pressure infrared spectrum from HgSe CQD with an intraband peak energy at $2500 \mathrm{~cm}^{-1}$. e and frespectively TEM image and zero pressure infrared spectrum from $\mathrm{HgSe}$ CQD with an intraband peak energy at $1000 \mathrm{~cm}^{-1}$ 


\section{Material characterization}

Under high pressure, the zinc blende phase of the HgTe nanocrystal switches toward a cinnabar phase, which refined diffractogram is provided in Figure $\mathrm{S} 3 \mathrm{a}$. A scheme of the cinnabar HgTe unit cell is given in Figure $\mathrm{S} 3 \mathrm{~b}$. We found the structural parameter of the hexagonal phase of $\mathrm{HgTe}$ to $\mathrm{be} a=b=0.447 \mathrm{~nm}$ and $\mathrm{c}=0.943 \mathrm{~nm}$ under $5.5 \mathrm{GPa}$ of pressure.
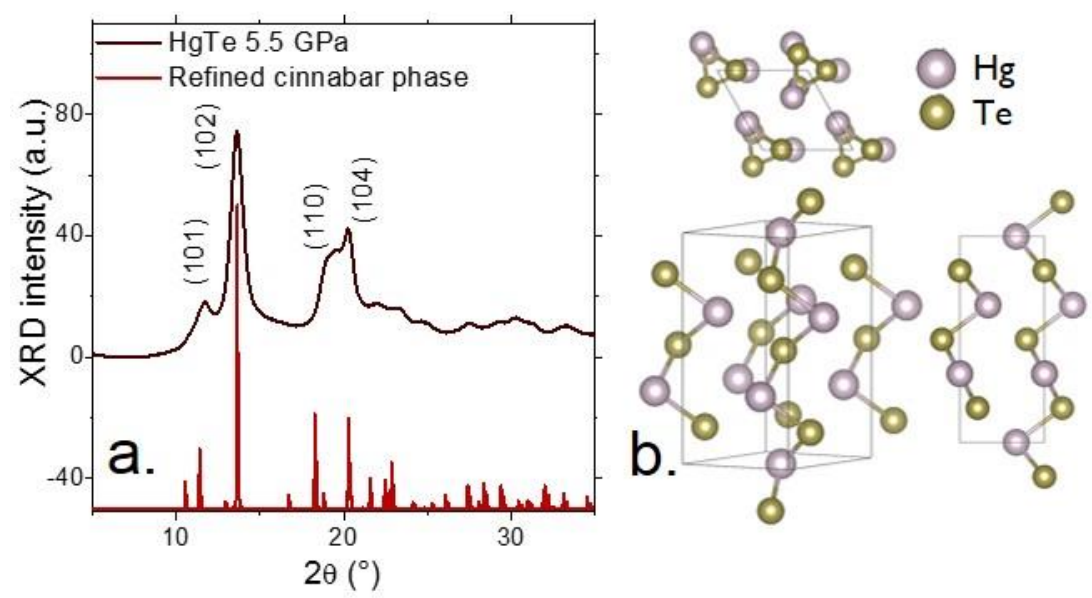

Figure $S 3$ a. Experimental and refined X-ray diffractogram from HgTe nanocrystal under a 5.5 GPa pressure. b. Scheme of the HgTe cinnabar unit cell. 


\section{IR spectroscopy under pressure}

\subsection{Signal filtering}

The infrared data obtained from the setup described in figure 2 of the main text present oscillations, see Figure $\mathrm{S} 4$. They result from interference with the diamond cell. The high frequency oscillations are then removed using an FFT filtering procedure, see Figure $\mathrm{S} 4$.
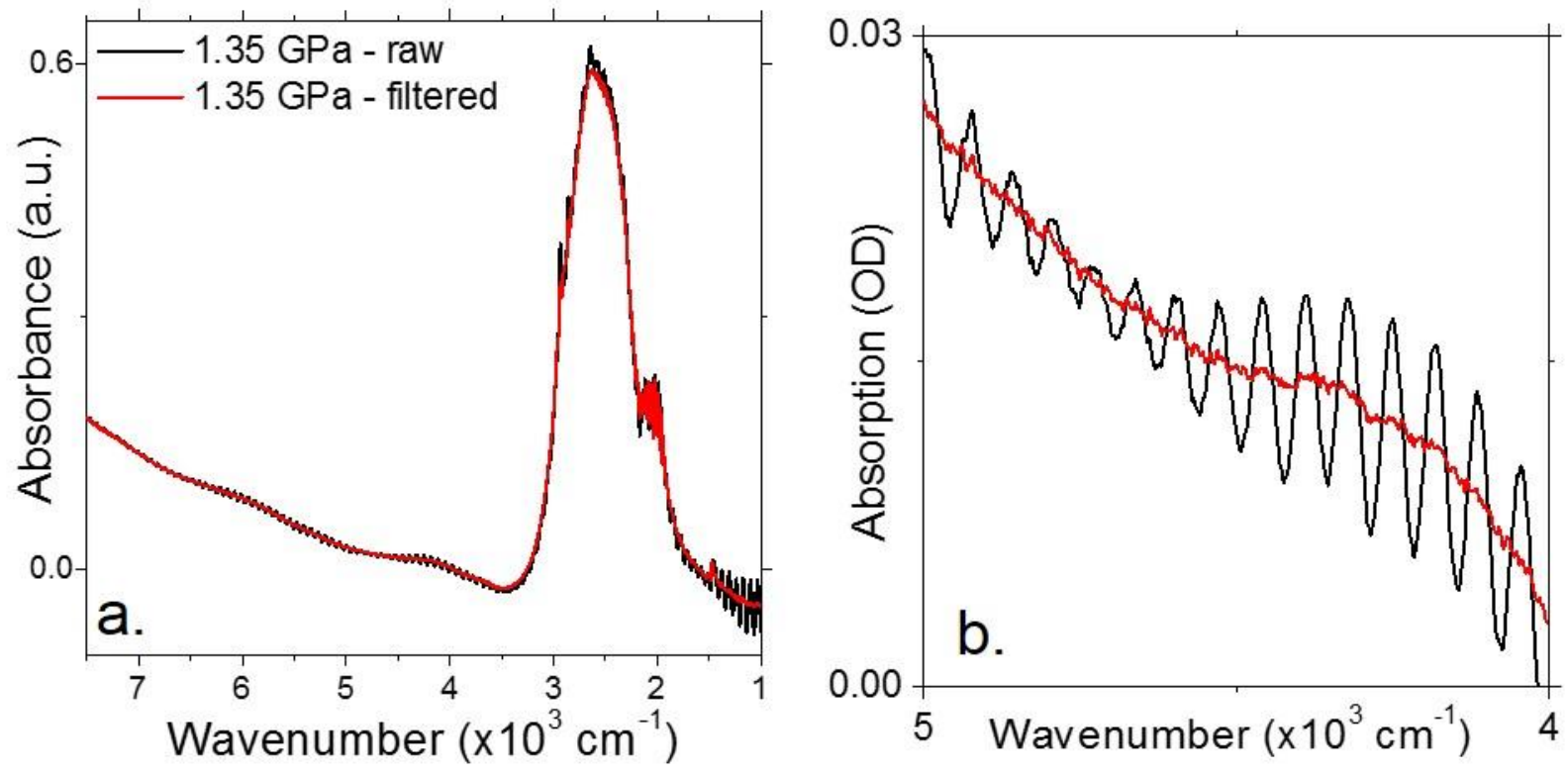

Figure $S 4$ a raw and filtered infrared spectrum obtained from HgSe CQD with intraband peak energy at $2500 \mathrm{~cm}^{-1} \cdot b$ is a zoom on the same spectrum in the range of energy between 4000 and $5000 \mathrm{~cm}^{-1}$.

4.2. IR spectroscopy under high pressure ( $P>3 \mathrm{GPa})$
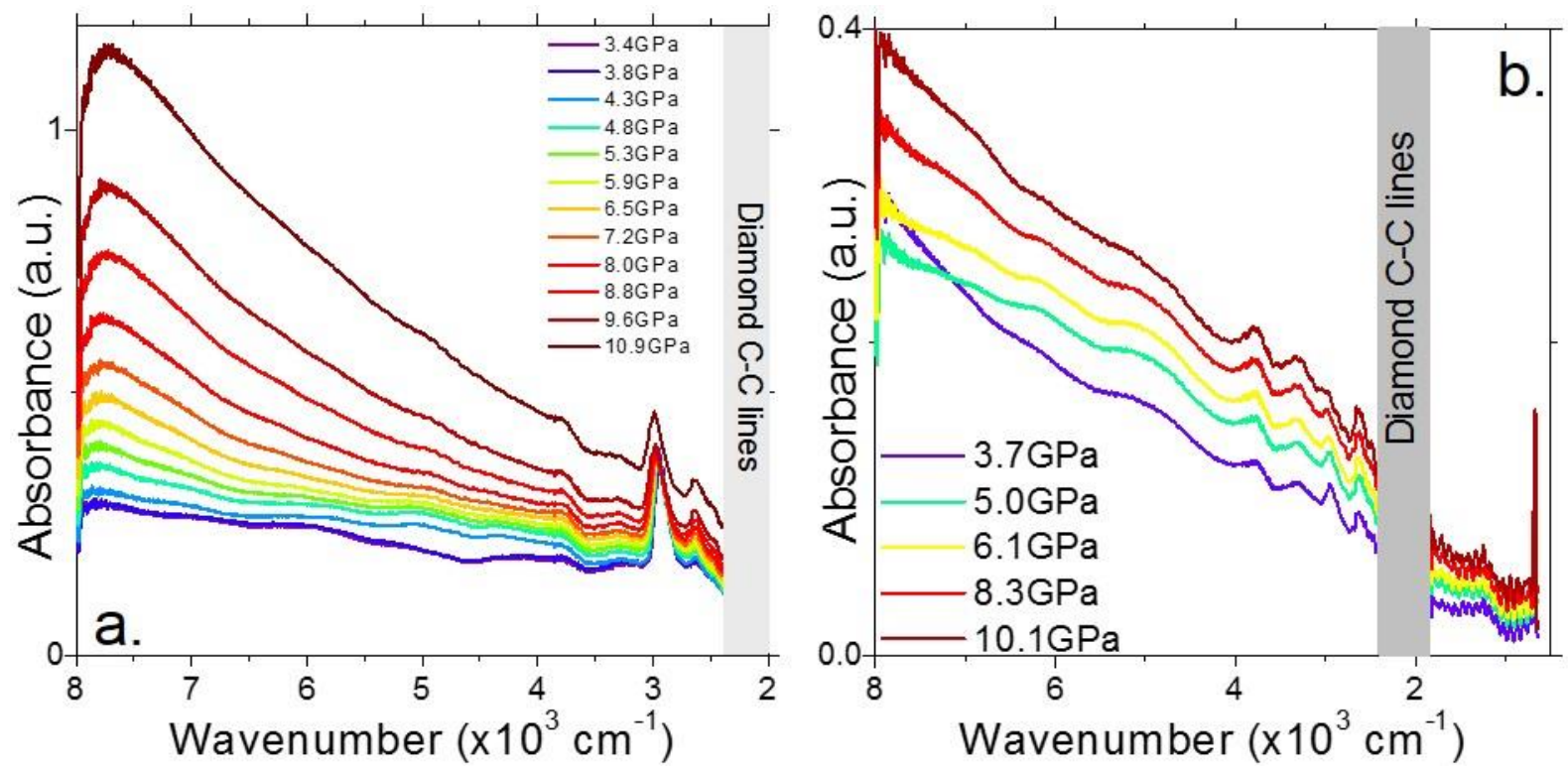

Figure $S 5$ a. Infrared spectra for HgTe CQD, with a $6000 \mathrm{~cm}^{-1}$ band edge at zero pressure, in the 3 to 10 GPa range of pressure. $b$ Infrared spectra for HgSe CQD, with a $1000 \mathrm{~cm}^{-1}$ intraband peak at zero pressure, in the 3 to $10 \mathrm{GPa}$ range of pressure. 


\section{5. k.p simulation}

The energy dispersion $E(k)$ with wavevector $\mathrm{k}$ in the Brillouin zone is calculated using a Pidgeon-Brown 8 band k.p formalism as show in Figure $S 6 a .{ }^{1}$ The bulk states at the $\mathbf{k}$ vector are developed on a limited set consisting in 8 zone center Bloch states of $\Gamma_{6}, \Gamma_{7}$ and $\Gamma_{8}$ symmetry. Through adjustable parameters the $8 \times 8$ matrix accounts for the k.p interaction between these states but also for the perturbative influence of the zone center states outside of the basis set. These k.p parameters are chosen to reproduce as closely as possible the band structure given in the figure 3(c) from Ref. ${ }^{2}$ obtained through the h-QSGW scheme of Svane et al. Note that on purpose we do not introduce the linear term in the k.p matrix that leads to the splitting of the heavy $\Gamma_{8}$ band along $\Gamma-L$ obtained by Svane et al since this splitting plays a negligible contribution to the interband energy. The obtained k.p dispersions $E(k)$ of the 8 bands are given Figure $\mathrm{S} 6 \mathrm{a}$. It is plotted using the same scale as $\operatorname{Ref}^{2}$ in order to be readily compared.

The dispersion of the two $\Gamma_{8}$ bands can be approximated near the Brillouin zone center through the Dresselhaus-KipKittel formula ${ }^{3} \quad E(k)=E(k=0)+A k^{2} \pm \sqrt{B^{2} k^{4}+C^{2}\left(k_{x}^{2} k_{y}^{2}+k_{y}^{2} k_{z}^{2}+k_{z}^{2} k_{x}^{2}\right)}$, with $\mathrm{A}=37.8 \hbar^{2} / 2 / m_{0}, \quad \mathrm{~B}=-$ $20.39 \hbar^{2} / 2 / m_{0}, \mathrm{C}=-107.8 \hbar^{2} / 2 / m_{0}$ obtained from the k.p hamiltonian. $\hbar=h / 2 / \pi$ is the reduced Plank constant and $m_{0}$ is the electron mass. This formula amounts to a parabolic dispersion along each direction of the Brillouin zone. We use this parabolic dispersion to evidence the strong non parabolicity of $E(k)$ a few percent away from the Brillouin zone in the comparison of Figure $5 \mathrm{a}$.

In semiconductors exhibiting a cubic structure, a hydrostatic pressure $P$ produces a scalar deformation tensor $\epsilon$ so that for the diagonal elements $\epsilon_{x x}=\epsilon_{y y}=\epsilon_{z z}=P / 3 / B$ where $B$ is the bulk modulus and $\epsilon_{x y}=\epsilon_{y z}=\epsilon_{z x}=0$ for the shear strain component. ${ }^{4}$ Since the contraction of the lattice parameter produces an expansion of the Brillouin zone and simultaneously an expansion of the wavevectors $k$ involved in the confined states, we neglect these two roughly self-cancelling effects in the strained electronic structure calculations. The effect on strain on the energy dispersion is accounted for through the change in the energy difference between $\Gamma_{6}$ and $\Gamma_{8}$ state energies at the zone center with a deformation potential of $d\left(E_{\Gamma_{6}}-E_{\Gamma_{8}}\right) / d \epsilon / 3=-2.4 \mathrm{eV}$. As depicted in Figure $\mathrm{S} 6 \mathrm{~b}$ and $5 \mathrm{a}$, the pressure first results in a decrease of the conduction band effective mass $m_{e}^{*}(k=0, P)$, until $\epsilon=-2.2 \%$ where the band gap opens and the effective mass increases again. The equivalent mass $m_{e}^{*}(k, P)$ calculated at $k=10 \% 2 \pi / a$ along the $\Gamma-L$ direction follows the same trend but with much less amplitude variation.
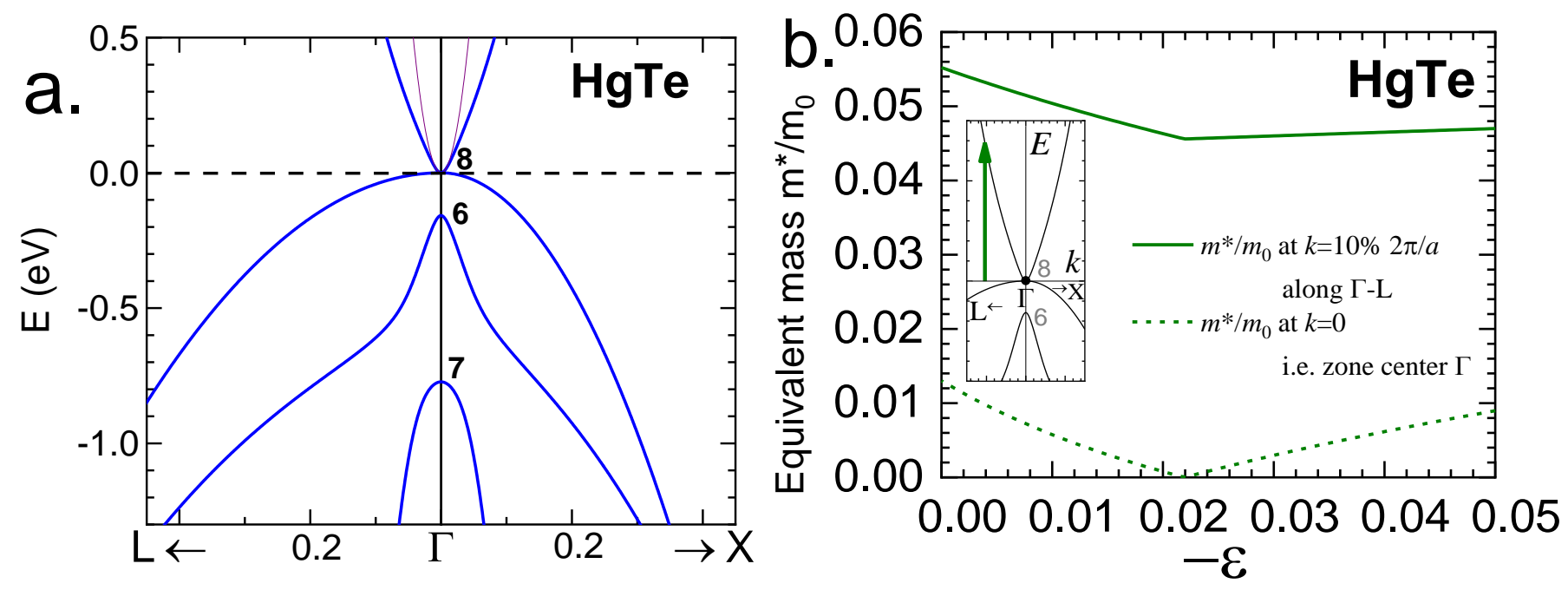

Figure $\mathbf{S} 6$ Simulated band structure of HgTe at zero strain from $\boldsymbol{k} \cdot \boldsymbol{p}$ simulation. Parameters are obtained by fitting the predicted band structure by Svane et $a l^{2}{ }^{2} . b$. Equivalent mass of the conduction band as a function of strain $\epsilon$, at the zone center and at $10 \%$ of the Brillouin zone along $\Gamma-L$. The equivalent mass is the mass $m^{*}$ that gives the energy $E$ of the conduction band at $\boldsymbol{k}$ point through the formula $E=E(k=0)+\frac{\hbar^{2} k^{2}}{2 m^{*}}$. 


\section{References}

(1) Pidgeon, C. R.; Brown, R. N. Phys. Rev. 1966, 146, 575-583.

(2) Svane, A.; Christensen, N. E.; Cardona, M.; Chantis, A. N.; van Schilfgaarde, M.; Kotani, T. Phys. Rev. B 2011, 84, 205205.

(3) Dresselhaus, G.; Kip, A. F.; Kittel, C. Phys. Rev. 1955, 98, 368-384.

(4) YU, P.; Cardona, M. Fundamentals of Semiconductors: Physics and Materials Properties, 4th ed.; Graduate Texts in Physics; Springer-Verlag: Berlin Heidelberg, 2010. 